\title{
Association between fixed orthodontic treatment and dental caries: a 1-year longitudinal study
}

\author{
Alice Souza PINTO(a) (iD \\ Luana Severo ALVES(b) iD \\ Marisa MALTZ(c) iD \\ Júlio Eduardo do Amaral \\ ZENKNER(d) iD $^{(\mathrm{d})}$ \\ (a) Universidade Franciscana - UFN, School of \\ Dentistry, Santa Maria, RS, Brazil. \\ (b) Universidade Federal de Santa Maria - \\ UFSM, School of Dentistry, Department \\ of Restorative Dentistry, Santa Maria, \\ RS, Brazil. \\ (c) Universidade Federal do Rio Grande do \\ Sul - UFRGS, Faculty of Odontology, \\ Department of Social and Preventive \\ Dentistry, Porto Alegre, RS, Brazil. \\ (d) Universidade Federal de Santa Maria - \\ UFSM, School of Dentistry, Department of \\ Stomatology, Santa Maria, RS, Brazil.
}

Declaration of Interests: The authors certify that they have no commercial or associative interest that represents a conflict of interest in connection with the manuscript.

Corresponding Author:

Luana Severo Alves

E-mail: luanaseal@gmail.com

Submitted: March 11, 2020

Accepted for publication: July 27, 2020

Last revision: August 19, 2020

\begin{abstract}
This longitudinal study aimed to assess the association between the use of fixed orthodontic appliances and the incidence/increment of active caries lesions in adolescents and young adults over a one-year period. A total of 135 10-30-year-old individuals were divided into two groups: Group G0 was composed of individuals who required orthodontic treatment, but who did not undergo fixed orthodontic therapy over the study period $(n=70)$; Group G1 was composed of individuals who used a fixed orthodontic appliance for 1 year $(n=65)$. Data collection included a questionnaire and clinical oral examinations (plaque index, gingival index, and dental caries index), performed at baseline and after 1 year. Caries examination was performed by a single calibrated examiner, after tooth cleaning and drying, and included the recording of non-cavitated and cavitated, inactive and active lesions of all tooth surfaces. Poisson regression models were used to assess the association between the group and the following study outcomes: incidence (binary variable) and increment (counting variable). The incidence of active caries lesions was $4.8 \%$ in G0 and $39.6 \%$ in G1. The mean increment of active caries lesions was 0.14 in G0 and 0.61 in G1. G1 showed a greater risk of developing active caries lesions than G0 (incidence analysis, adjusted $\mathrm{IRR}=9.48$, 95\%CI=2.62-34.30; increment analysis, adjusted $\mathrm{IRR}=4.13$; 95\% CI $=1.94-8.79$ ). In conclusion, this study showed that individuals undergoing fixed orthodontic therapy for 1 year had a significantly higher incidence and increment of active caries lesions than those without a fixed appliance.
\end{abstract}

Keywords: Dental Caries; Orthodontic Appliances; Incidence; Longitudinal Studies.

\section{Introduction}

Despite the substantial reduction in caries prevalence and severity observed worldwide in the last decades, ${ }^{1,2}$ dental caries remains a public health challenge. ${ }^{3}$ In 2010 , it was the most prevalent health condition, affecting $35 \%$ of the global population, or 2.4 billion people worldwide. ${ }^{4}$ Considering its multifactorial etiology, different factors may contribute to a higher risk of developing the disease. In this sense, the use of fixed orthodontic appliances has been suggested as a risk 
factor for dental caries, since it promotes favorable conditions for biofilm accumulation and hampers the performance of usual oral hygiene procedures. ${ }^{5}$ In addition, the irregular surfaces of the brackets, bands, wires, and other attachments can limit the natural self-cleaning mechanisms of the oral musculature and saliva. ${ }^{5}$ A recent systematic review with meta-analysis summarized the prevalence and incidence rates of non-cavitated caries lesions (NCL) during orthodontic treatment. ${ }^{5} \mathrm{~A}$ total of 14 studies published up to March 2015 were included. The authors found a mean prevalence of $68.4 \%$ (data derived from 9 studies) and a mean incidence of $45.8 \%$ (data derived from 7 studies). In addition, the authors concluded that certain factors were found to increase the incidence of NCL, such as poor oral hygiene, younger age of the patient, male patients, and duration of the treatment.

An important limitation of the longitudinal studies available in the literature is the method of detecting caries during orthodontic therapy. The occurrence of NCL in several studies was investigated by clinical photographs taken before and after orthodontic treatment for documentary purposes. . $7,7,8,9,10,11,12^{\text {To }}$ the best of our knowledge, there is no longitudinal study adopting the appropriate clinical criteria for detection of NCL among orthodontic patients. A previous cross-sectional study by our research group investigated caries activity among orthodontic patients using clinical examinations taken after tooth cleaning and drying. ${ }^{13}$ The authors showed that the longer the duration of the orthodontic treatment, the higher the prevalence/extent of active caries lesions. However, only longitudinal studies may provide evidence of causality due to the temporal component. ${ }^{14}$

This longitudinal study aimed to assess the association between the use of fixed orthodontic appliances and the incidence/increment of active caries lesions in adolescents and young adults over a one-year period.

\section{Methodology}

\section{Study design and sample}

This longitudinal study was conducted in Santa Maria, South Brazil, and included patients aged
10-30 years, who sought orthodontic treatment in an orthodontic graduate program. The city has been supplied with fluoridated water for more than 30 years (0.7-0.8 ppm F). Individuals were divided into two groups, as follows: Group G0, composed of individuals who required orthodontic treatment, but who did not undergo fixed orthodontic therapy over the study period; and Group G1, composed of individuals who used a fixed orthodontic appliance for 1 year (10-14 months of treatment).

Sample size calculation considered the following parameters: difference of $20 \%$ in the incidence of active caries lesions between the comparative groups, power of $80 \%$ and confidence interval of $95 \%$. This resulted in a required sample size of 65 individuals per group. Patients who had congenital anomalies, special needs, or were taking systemic medication to treat chronic diseases that could interfere with caries activity were excluded from the sample. In addition, patients requiring traction of impacted teeth and repositioning of widely lingual/buccal teeth (> $2 \mathrm{~mm}$ ) were not included.

Fixed corrective orthodontic treatment in G1 participants was carried out using conventional metal brackets, straight wire technique, and orthodontic arches fixed with simple elastic bands. Orthodontic rings (bands) were adapted to the molars with glass ionomer cement. Although G1 patients underwent an individualized caries control program to control caries activity (oral hygiene instruction, diet counseling, topical fluoride application) before installation of the orthodontic appliance, no specific program to control caries development was provided to the patients during the orthodontic treatment.

The study protocol was approved by the Research Ethics Committee of the Federal University of Santa Maria (protocol number 0109/2013). All patients or their legal guardians signed a written informed consent form. The study was conducted according to the Declaration of Helsinki.

\section{Data collection}

Initially, the subjects answered a questionnaire on sociodemographic characteristics (age, sex, mother's education, and socioeconomic status) and oral hygiene habits (tooth brushing and dental floss). Clinical 
examinations were conducted in a dental unit, with a clinical mirror and a WHO probe. First, the plaque index and the gingival index were recorded on four surfaces per tooth, using the 4-score system originally proposed. ${ }^{15}$ The plaque index was recorded as follows: (0) no plaque in the gingival area; (1) a plaque biofilm adhering to the free gingival margin, recognized only by running a probe across the tooth surface; (2) moderate accumulation of soft deposits on the gingival margin, visible to the naked eye; or (3) abundance of soft matter on the gingival margin..$^{15}$ In regard to the gingival index, surfaces were classified as: (0) normal gingiva; (1) mild inflammation, slight change in color, slight edema, no bleeding on probing; (2) moderate inflammation, redness, edema and glazing, bleeding on probing; or (3) severe inflammation, marked redness, edema, ulceration, tendency of spontaneous bleeding. ${ }^{15}$

Afterwards, professional prophylaxis was performed with sodium bicarbonate spray (Jetlaxis Uno, Schuster, Santa Maria, Brazil), tooth surfaces were dried with an air-water syringe and isolated with cotton rolls, and the presence and activity of caries lesions was assessed according to a previously used index. ${ }^{16}$ All tooth surfaces were examined and classified according to their clinical characteristics (surface texture and brightness). Active non-cavitated lesion was defined as an opaque enamel with a dull whitish surface; inactive non-cavitated lesion was defined as a shiny appearance of the surface area, with white or different degrees of brownish discoloration; active cavitated lesion was defined as a localized surface destruction with active characteristics (dull whitish enamel and soft dentin of light brown color); inactive cavitated lesion was defined as a localized surface destruction with arrested characteristics (shiny, hard surfaces with different degrees of brownish discoloration). Filled and missed surfaces were also recorded. Clinical examinations were performed at baseline (in G1, immediately before installation of the fixed appliance) and after 1 year, based on the same protocol.

\section{Reproducibility}

All the clinical examinations were conducted by a single examiner (ASP). After intensive training sessions supervised by a reference examiner (JEAZ), the first round of repeated examinations was performed on 10 individuals without any orthodontic appliance. An intraexaminer kappa value (ASPxASP) of 0.92 and an interexaminer kappa value (ASPxJEAZ) of 0.87 were obtained. Examiner reproducibility was assessed after the second round of repeated examinations on 10 individuals using a fixed orthodontic appliance, in which case an intraexaminer kappa value (ASPxASP) of 0.89 was obtained. In both rounds, double examinations were performed on individuals not pertaining to the study sample, after a minimal time interval of 7 days. Considering that plaque accumulation and gingival bleeding are variable conditions, examiner calibration was not assessed for these indexes.

\section{Data analysis}

The main outcomes of this study were the incidence and increment of caries activity. The incidence of caries activity was defined as the proportion of individuals who developed at least one active caries lesions (either non-cavitated or cavitated) over the study period. The increment of caries activity was defined as the difference between the number of active lesions at follow-up and the number of active lesions at baseline.

The independent variables evaluated were age, sex, mother's education, socioeconomic status, family income, tooth brushing frequency, dental floss, plaque index, and gingival index. Subjects were classified into three age groups: $\leq 15$ years, $16-20$ years, $>20$ years. Mother's education was classified as primary school, high school, and university. Socioeconomic status was collected as a 4-category variable (high, mid-high, mid-low, and low), and grouped to compose a binary variable: high/mid-high and mid-low/low. Family income was classified into $\leq 3$ and $>3$ Brazilian minimum wages (1 BMW corresponded to about 295 US dollars during the period of data collection). Tooth brushing frequency was dichotomized as $\leq 2$ times/day and $\geq 3$ times/day. The use of dental floss was classified as daily and non-daily. Plaque index and gingival index were calculated as the mean score of all evaluated sites, and maintained as a continuous variable. 
G0 and G1 were compared according to baseline characteristics, using the chi-square test (qualitative variables) and the Wald test (quantitative variables). Poisson regression models were used to assess the association between the group and the study outcomes: incidence (binary variable) and increment (counting variable). First, an unadjusted model was used to investigate the association between the group and the incidence/increment disregarding the influence of other factors. Then, an adjusted analysis was performed in order to account for the influence of important cofactors notably related to caries development. Age, sex, mother's education, socioeconomic status, family income, tooth brushing, dental floss, plaque index, and gingival index were included and maintained in the adjusted model irrespective of their $p$-values, in order to remove any possible effect of these variables on the associations found. Data analysis was performed using STATA software (Stata 14.2 for Windows; Stata Corporation, College Station, USA). The significance level was set at $5 \%$.

\section{Results}

A total of 70 individuals were included in Group 0 , whereas Group 1 was composed of 65 patients. Sample distribution according to baseline characteristics and group is presented in Table 1. G0 and G1 were similar in regard to all evaluated variables, with the exception of age. G1 presented a higher proportion of individuals aged $\leq 15$ years than G0. It is important to emphasize that the prevalence of caries activity, the number of active caries lesions, the DMFT, the plaque index, and the gingival index were similar in both groups at the baseline examination.

Of the 135 individuals included in the sample, 14 already presented caries activity at baseline (seven patients per group). Therefore, 121 participants were considered at risk for developing the disease, and were included in the incidence analyses. Incidence of caries activity by group and the Poisson regression models are shown in Table 2. A caries activity incidence of $4.8 \%$ was observed for G0, and 39.6\%, for G1. Individuals undergoing fixed orthodontic treatment showed an 8 -fold greater risk of developing at least one active caries lesions, compared with individuals without a fixed appliance (unadjusted IRR $=8.33 ; 95 \% \mathrm{CI}=2.50$ 27.73). After the inclusion of sociodemographic, behavioral, and clinical variables in the adjusted model, this estimate showed a slight increase (adjusted $\mathrm{IRR}=9.48 ; 95 \% \mathrm{CI}=2.62-34.30$ ).

The number of active caries lesions developed over the study period ranged from 0 to 6 , as shown in Figure. The most commonly affected surface was

Table 1. Sample distribution according to baseline characteristics and group.

\begin{tabular}{|c|c|c|c|}
\hline \multirow{2}{*}{ Variable } & Group 0 & Group 1 & \multirow{2}{*}{$p$-value** } \\
\hline & n (\%) & n (\%) & \\
\hline \multicolumn{4}{|l|}{ Age (years) } \\
\hline$\leq 15$ & $19(27.1)$ & $35(53.8)$ & \\
\hline $16-20$ & $26(37.1)$ & $14(21.5)$ & \\
\hline$>20$ & $25(35.7)$ & $16(24.6)$ & 0.006 \\
\hline \multicolumn{4}{|l|}{ Sex } \\
\hline Male & $28(40.0)$ & $27(41.5)$ & \\
\hline Female & $42(60.0)$ & $38(58.5)$ & 0.86 \\
\hline \multicolumn{4}{|l|}{ Mother's education" } \\
\hline Primary school & $27(39.1)$ & $25(38.5)$ & \\
\hline High school & $24(34.8)$ & $19(29.2)$ & \\
\hline University & $18(26.1)$ & $21(32.3)$ & 0.68 \\
\hline \multicolumn{4}{|l|}{ Socioeconomic status } \\
\hline High/Mid-high & $37(52.9)$ & $36(55.4)$ & \\
\hline Mid-low/Low & $33(47.1)$ & $29(44.6)$ & 0.77 \\
\hline \multicolumn{4}{|l|}{ Family income } \\
\hline$\leq 3 \mathrm{BMW}$ & $46(65.7)$ & $36(55.4)$ & \\
\hline$>3 \mathrm{BMW}$ & $24(34.3)$ & $29(44.6)$ & 0.22 \\
\hline \multicolumn{4}{|l|}{ Tooth brushing } \\
\hline$\leq 2$ times/day & $27(38.6)$ & $16(24.6)$ & \\
\hline$\geq 3$ times/day & $43(61.4)$ & $49(75.4)$ & 0.08 \\
\hline \multicolumn{4}{|l|}{ Dental floss* } \\
\hline Non-daily & $40(58.0)$ & $31(47.7)$ & \\
\hline Daily & $29(42.0)$ & $34(52.3)$ & 0.23 \\
\hline \multicolumn{4}{|c|}{ Prevalence of active caries } \\
\hline 0 active caries & $63(90.0)$ & $58(89.2)$ & \\
\hline \multirow[t]{2}{*}{$\geq 1$ active caries } & 7 (10.0) & $7(10.8)$ & 0.88 \\
\hline & Mean $( \pm \mathrm{SD})$ & Mean $( \pm S D)$ & $p$-value ${ }^{* * *}$ \\
\hline Active caries lesions & $0.24(0.94)$ & $0.31(0.63)$ & 0.64 \\
\hline DMFT (cavity level) & $3.99(3.42)$ & $3.55(3.32)$ & 0.46 \\
\hline Plaque index & $0.20(0.16)$ & $0.22(0.18)$ & 0.62 \\
\hline Gingival index & $0.20(0.19)$ & $0.20(0.21)$ & 0.97 \\
\hline
\end{tabular}

BMW: Brazilian minimum wage (equivalent to around 295 US dollars during data gathering); DMFT: decayed, missing, and filled teeth; SD: standard deviation; *Missing value. ${ }^{* *}$ Chi-square test; "Wald test. 
Table 2. Incidence of caries activity over the study period and its association with independent variables. Unadjusted and adjusted Poisson regression models $(\mathrm{n}=121)$.

\begin{tabular}{lccccc}
\hline \multirow{2}{*}{ Group } & Incidence & \multicolumn{2}{c}{ Unadjusted } & \multicolumn{2}{c}{ Adjusted* } \\
\cline { 2 - 4 } & $\%(95 \% \mathrm{Cl})$ & IRR $(95 \% \mathrm{Cl})$ & $\mathrm{p}$-value & IRR $(95 \% \mathrm{Cl})$ & $\mathrm{p}$-value \\
\hline 0 & $4.8(-0.6-10.1)$ & 1.00 & & 1.00 & \\
1 & $39.6(26.8-52.5)$ & $8.33(2.50-27.73)$ & 0.001 & $9.48(2.62-34.30)$ & 0.001 \\
\hline
\end{tabular}

*Estimates have been adjusted for age, sex, mother's education, socioeconomic status, family income, tooth brushing, dental floss, plaque index, and gingival index; IRR: incidence risk ratio; $\mathrm{Cl}$ : confidence interval.

buccal (62\%), followed by approximal (28\%), lingual $(5 \%)$, and occlusal (4\%). Table 3 presents the increment of active caries lesions according to group, and the risk assessment analysis. Individuals without a fixed orthodontic appliance presented a mean increment of active lesions of 0.14 , whereas those using an appliance developed an average of 0.61 new active lesions. Poisson regression models showed that individuals composing G1 presented approximately a 4-fold higher risk of developing an additional active lesion than participants in G0 (unadjusted IRR $=4.31$; $95 \% \mathrm{CI}=2.15-8.61$; adjusted $\mathrm{IRR}=4.13 ; 1.94-8.79)$.

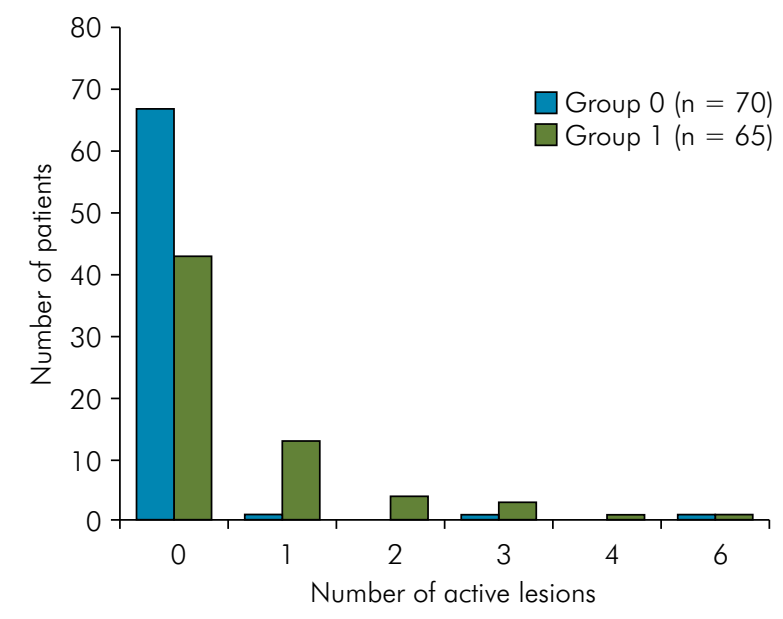

Figure. Number of active caries lesions developed over the study period.

\section{Discussion}

This study assessed the association between the use of fixed orthodontic appliances and the occurrence of active caries lesions over a one-year period. A significantly greater risk of caries development was observed among patients submitted to fixed orthodontic therapy, compared with individuals without the appliance. This is the first longitudinal study to assess this relationship using the appropriate clinical criteria and conditions for detecting noncavitated caries lesions in both observational periods (baseline and follow-up).

Previous studies have demonstrated a high caries incidence among orthodontic patients when clinical photographs are used as the diagnostic tool. $.7,7,9,10,11,12$ Although Chapman et al. ${ }^{11}$ reported that these photographs are a valid method for examining the percentage of buccal surfaces affected by NCL, there is no validation study comparing this method of detection with conventional clinical examinations. Considering that photographs for orthodontic documentation are usually taken without cleaning and drying of tooth surfaces, this method can be presumed to underestimate the number of initial caries lesions in enamel, as previously described in the literature. ${ }^{17}$ Although the purpose of these studies has been limited to the evaluation of caries on buccal

Table 3. Increment of caries activity over the study period and its association with independent variables. Unadjusted and adjusted Poisson regression models $(n=135)$.

\begin{tabular}{lccccc}
\hline \multirow{2}{*}{ Group } & Increment & \multicolumn{2}{c}{ Unadjusted } & \multicolumn{2}{c}{ Adjusted* $^{*}$} \\
\cline { 2 - 4 } & Mean $(95 \% \mathrm{Cl})$ & IRR $(95 \% \mathrm{Cl})$ & $\mathrm{p}$-value & IRR $(95 \% \mathrm{Cl})$ & $\mathrm{p}$-value \\
\hline 0 & $0.14(-0.05-0.33)$ & 1.00 & & 1.00 & \\
1 & $0.61(0.33-0.89)$ & $4.31(2.15-8.61)$ & $<0.001$ & $4.13(1.94-8.79)$ & $<0.001$ \\
\hline
\end{tabular}

*Estimates have been adjusted for age, sex, mother's education, socioeconomic status, family income, tooth brushing, dental floss, plaque index, and gingival index; IRR: incidence risk ratio; $\mathrm{Cl}$ : confidence interval. 
surfaces associated with orthodontic brackets, it is well known that their findings do not express the overall situation of the patient in regard to caries activity. The rigorous clinical examination protocol of the present study, including tooth cleaning and drying and evaluation of all tooth surfaces, corroborated the previous literature. The $39.6 \%$ incidence of active caries observed among users of fixed orthodontic appliance was similar to the $45.8 \%$ incidence rate found in a recent meta-analysis ${ }^{5}$. Considering this finding, it is possible to infer that the different methods to evaluate dental caries (clinical examination or photographs) may have had no major impact on the incidence estimates. Although this population has access to public water fluoridation and more than half reported brushing teeth with fluoridated toothpaste $\geq 3$ times/day, individuals showed a high incidence of active lesions during orthodontic treatment. The risk assessment analysis found that using a fixed orthodontic appliance afforded an 8-9-fold higher risk of developing at least one active caries lesion in a period of 1 year, in comparison with the group without the fixed appliance. In order to remove the effect of possible confounders, the adjusted analysis included all the other independent variables in the model. The adjusted estimate was slightly higher than the crude estimate, thus reinforcing the effect of using an orthodontic appliance in the association.

In addition to the incidence analysis, the increment of active caries lesions was also evaluated. A greater caries increment was found in G1 than in G0, in agreement with the incidence data. In the risk assessment analysis, the use of a fixed orthodontic appliance resulted in an approximately 4 -fold greater risk of individuals with the appliance developing an additional active lesion than individuals without it, even after adjusting for possible confounding factors. There is no previous study in the literature evaluating caries increment in orthodontic patients.

The noteworthy strengths of our study include its clinical examination protocol and the high reproducibility of the examiner. Furthermore, our study was able to estimate the effect of a fixed orthodontic treatment on caries activity more accurately over a one-year period by using risk assessment analysis, adjusted for sociodemographic, behavioral, and intraoral factors. Considering the number of individuals included in previous studies using photographs, ${ }^{6,79,10,12}$ a possible limitation of our study might be its sample size. Notwithstanding, the number of patients examined in our study gave it sufficient statistical power to detect the differences under study between the groups. The similarities between the comparative groups were checked at baseline, and a significant difference was detected in regard to age. We acknowledge that age may affect the oral hygiene pattern of subjects, including skills and motivation. Older individuals tend to practice better oral hygiene than younger ones, ${ }^{18}$ and this has been found to affect the occurrence of caries among orthodontic patients. ${ }^{6,7}$ Since the existence of even a slight age difference between the comparative groups could impact the outcome, the "age" factor was included in the adjusted models. It could be argued that the lack of data on the type of malocclusion of the included sample could have some effect on the study outcomes. However, considering that G1 patients received orthodontic movements of tooth alignment and leveling in the first months of treatment, and that G0 patients had problems that persisted throughout the study period, we can speculate that the effect of the orthodontic appliance on caries occurrence in G1 may have surpassed the possible effect of malocclusion on caries occurrence in $\mathrm{G} 0 .{ }^{19}$ Lastly, another possible limitation of our study is the lack of data on sugar consumption, given its role in caries development. ${ }^{20}$

\section{Conclusion}

This longitudinal study showed that individuals undergoing fixed orthodontic therapy over a one-year period had a significantly higher incidence and increment of active caries lesions than those without a fixed appliance. These findings may shed light on the issue of caries activity among orthodontic patients, and underscore the need for monitoring caries activity during fixed orthodontic therapy.

\section{Acknowledgments}

We would like to thank all the participating patients and their legal guardians for collaborating on the study. Conflicts of interest: none to declare. 


\section{References}

1. Beltrán-Aguilar ED, Estupiñán-Day S, Báez R. Analysis of prevalence and trends of dental caries in the Americas between the 1970s and 1990s. Int Dent J. 1999 Dec;49(6):322-9. https://doi.org/10.1111/j.1875-595X.1999.tb00532.x

2. Cleaton-Jones P, Fatti P, Bönecker M. Dental caries trends in 5- to 6-year-old and 11- to 13-year-old children in three UNICEF designated regions - Sub Saharan Africa, Middle East and North Africa, Latin America and Caribbean: 1970-2004. Int Dent J. 2006 Oct;56(5):294-300. https://doi.org/10.1111/j.1875-595X.2006.tb00104.x

3. Peres MA, Macpherson LM, Weyant RJ, Daly B, Venturelli R, Mathur MR, et al. Oral diseases: a global public health challenge. Lancet. 2019 Jul;394(10194):249-60. https://doi.org/10.1016/S0140-6736(19)31146-8

4. Kassebaum NJ, Bernabé E, Dahiya M, Bhandari B, Murray CJ, Marcenes W. Global burden of untreated caries: a systematic review and metaregression. J Dent Res. 2015 May;94(5):650-8. https://doi.org/10.1177/0022034515573272

5. Sundararaj D, Venkatachalapathy S, Tandon A, Pereira A. Critical evaluation of incidence and prevalence of white spot lesions during fixed orthodontic appliance treatment: A meta-analysis. J Int Soc Prev Community Dent. 2015 Nov-Dec;5(6):433-9. https://doi.org/10.4103/2231-0762.167719

6. Akin M, Tazcan M, lleri Z, Basciftci FA. Incidence of white spot lesion during fixed orthodontic treatment. Turk J Orthod. 2013;26(2):98-102. https://doi.org/10.13076/j.tjo.2013.26.02_98

7. Richter AE, Arruda AO, Peters MC, Sohn W. Incidence of caries lesions among patients treated with comprehensive orthodontics. Am J Orthod Dentofacial Orthop. 2011 May;139(5):657-64. https://doi.org/10.1016/i.ajodo.2009.06.037

8. Khalaf K. Factors affecting the formation, severity and location of white spot lesions during orthodontic treatment with fixed appliances. J Oral Maxillofac Res. 2014 Apr;5(1):e4. https://doi.org/10.5037/jomr.2014.5104

9. Julien KC, Buschang PH, Campbell PM. Prevalence of white spot lesion formation during orthodontic treatment. Angle Orthod. 2013 Jul;83(4):641-7. https://doi.org/10.2319/071712-584.1

10. Enaia M, Bock N, Ruf S. White-spot lesions during multibracket appliance treatment: A challenge for clinical excellence. Am J Orthod Dentofacial Orthop. 2011 Jul;140(1):e17-24. https://doi.org/10.1016/j.ajodo.2010.12.016

11. Chapman JA, Roberts WE, Eckert GJ, Kula KS, González-Cabezas C. Risk factors for incidence and severity of white spot lesions during treatment with fixed orthodontic appliances. Am J Orthod Dentofacial Orthop. 2010 Aug;138(2):188-94. https://doi.org/10.1016/i.ajodo.2008.10.019

12. Gorelick L, Geiger AM, Gwinnett AJ. Incidence of white spot formation after bonding and banding. Am J Orthod. 1982 Feb;81(2):93-8. https://doi.org/10.1016/0002-9416(82)90032-X

13. Pinto AS, Alves LS, Maltz M, Susin C, Zenkner JE. Does the duration of fixed orthodontic treatment affect caries activity among adolescents and young adults? Caries Res. 2018;52(6):463-7. https://doi.org/10.1159/000488209

14. Hill AB. The environment and disease: association or causation? Proc R Soc Med. 1965 May;58(5):295-300. https://doi.org/10.1177/003591576505800503

15. Löe H. The Gingival Index, the Plaque Index and the Retention Index Systems. J Periodontol. 1967 Nov-Dec;38(6):610-6. https://doi.org/10.1902/jop.1967.38.6.610

16. Maltz M, Silva BB, Carvalho DQ, Volkweis A. Results after two years of non-operative treatment of occlusal surface in children with high caries prevalence. Braz Dent J. 2003;14(1):48-54. https://doi.org/10.1590/S0103-64402003000100009

17. Assaf AV, Meneghim MC, Zanin L, Mialhe FL, Pereira AC, Ambrosano GM. Assessment of different methods for diagnosing dental caries in epidemiological surveys. Community Dent Oral Epidemiol. 2004 Dec;32(6):418-25. https://doi.org/10.1111/j.1600-0528.2004.00180.x

18. Kudirkaite I, Lopatiene K, Zubiene J, Saldunaite K. Age and gender influence on oral hygiene among adolescents with fixed orthodontic appliances. Stomatologija. 2016;18(2):61-5.

19. Sá-Pinto AC, Rego TM, Marques LS, Martins CC, Ramos-Jorge ML, Ramos-Jorge J. Association between malocclusion and dental caries in adolescents: a systematic review and meta-analysis. Eur Arch Paediatr Dent. 2018 Apr;19(2):73-82. https://doi.org/10.1007/s40368-018-0333-0

20. Sheiham A, James WP. Diet and dental caries: the pivotal role of free sugars reemphasized. J Dent Res. 2015 Oct;94(10):1341-7. https://doi.org/10.1177/0022034515590377 